\title{
Magnetically Multilayer Polysaccharide Membranes for Biomedical Applications
}

\author{
Sara Gil, ${ }^{\dagger, \ddagger, \S}$ Joana M. Silva, ${ }^{\dagger, \ddagger, \S}$ and João F. Mano ${ }^{*,+, \S}$
}

*3B’s Research Group - Biomaterials, Biodegradables and Biomimetics, University of Minho, Headquarters of the European Institute of Excellence of Tissue Engineering and Regenerative Medicine, Avepark - Parque de Ciência e Tecnologia, Zona Industrial da Gandra, 4805-017 Barco GMR, Portugal

${ }^{\S}$ ICVS/3B's - PT Government Associate Laboratory, Braga/Guimarães, Portugal

\section{Supporting Information}

\begin{abstract}
Self-standing nanocomposite films based on biopolymers and functional nanostructures have been widely used due to their potential applications as active elements in biomedical devices. The coupling between chitosan (CHI) and alginate (ALG) multilayered films and magnetic nanoparticles (MNPs) allowed to fabricate magnetic responsive freestanding membranes with a high structural control along the thickness, using the layer-by-layer ( $\mathrm{LbL}$ ) methodology. The mechanical characterization evidenced a trend for an increase of both Young modulus, and ultimate tensile strength with the inclusion of MNPs, or by crosslinking with genipin. Additionally, the multilayered membranes exhibited shape memory properties triggered by hydration. The in vitro biological performance studies showed that cells were more viable and adherent with higher proliferation rates when MNPs were included in the membranes. Our results suggested the potential of the developed magneto-active freestanding membranes for biomedical applications, such as in tissue engineering and biomedical applications.
\end{abstract}

KEYWORDS: layer-by-layer, nanoparticles, magnetic responsive, shape memory, nanobiomaterials, smart nanocomposites, tissue engineering

\section{INTRODUCTION}

The incorporation of nanoscale building blocks, with controlled and predetermined architecture, into active nanostructured substrates has gained increasing attention. However, in the vast majority of systems developed so far, nanomaterials are firmly anchored to supporting substrates, thus behaving as integrated macroscopic systems. ${ }^{1,2}$ The development of active structures whose properties are defined by the active nanoscale building blocks still remains a challenge. When combined with varied nanostructures, freestanding ultrathin polymeric films demonstrate an increasing potential toward biomedical applications, including termomechanical sensors, nanoactuators, chemical and biological sensors, and in drug delivery systems. ${ }^{3-7}$ In this work, we propose developing freestanding multilayered films where nanomaterials are partially decoupled from the supporting substrate.

Despite the different techniques available for the construction of functional ultrathin organized films, layer-by-layer (LbL) assembly still emerges as one of the most popular and flexible methodologies to create advanced materials with nanoand micrometer scale control. ${ }^{8,9}$ This methodology is very versatile and enables the use of different charged species, including biological molecules (polysaccharides, proteins, viruses, or DNA) ${ }^{6,9-11}$ and organic or inorganic materials. ${ }^{12,13}$ Among the polysaccharides that have been used, chitosan $(\mathrm{CHI})$ and alginate (ALG) received particular attention because of their potential to be assembled using $\mathrm{LbL}$ methodology stability in physiological conditions, and also their biocompatibility. ${ }^{14-16}$

With the simple adjustment of processing parameters such as temperature, $\mathrm{pH}$, charge density, polyelectrolyte concentration chemical or photo cross-linking, and ionic strength, films with a large variety of architectures and properties can be produced. $^{9-11,17-20}$ Besides simple coating, this technique has successfully allowed the development of materials with more complex structural arrangements such as capsules, ${ }^{21,22}$ membranes, $^{23-25}$ hollow tubes, ${ }^{26}$ and porous scaffolds. ${ }^{27,28}$ In particular, the fabrication of freestanding nanocomposite LbL films with the use of a sacrificial layer were first reported by Mamedov and Kotov in 2000. ${ }^{29}$ Using low surface energy substrates, it was shown that freestanding films could be simply produced from the detachment of multilayers from the template. $^{24,30,31}$

Despite the myriad of nanomaterials available, magnetic nanoparticles (MNPs) have been widely employed in several biomedical applications because of their innate magnetic characteristics. Nowadays, the application of magnetic fields in surgical and diagnostic procedures is a well-accepted

Received: July 10, 2015

Accepted: August 26, 2015 
approach that allows the remote control of microdevices implanted in the human body through magnetic actuation. ${ }^{32,33}$ The application of engineered gradient magnetic fields at the clinical level is currently being studied for targeting nanoobjects toward tumors and for positioning magnetic tip guidewires in coronary arteries. $\mathrm{LbL}$ assembly has recently been applied to the encapsulation in polymeric films of several functional nanostructures as magnetic nanoparticles, ${ }^{34}$ gold nanoparticles, and carbon nanotubes, thus imparting magnetic, optical, mechanical, or electronic properties to the final structures. $^{35,36}$ In this work, we propose to incorporate MNPs onto CHI-ALG multilayered freestanding membranes.

Superparamagnetic iron-oxide nanoparticles (SPIONs) are unique because of the combination of excellent magnetic properties and biocompatibility. These particular nanoparticles do not retain any residual magnetism upon removal of a magnetic field, and usually comprise a magnetite $\left(\mathrm{Fe}_{3} \mathrm{O}_{4}\right)$ or maghemite $\left(\gamma-\mathrm{Fe}_{2} \mathrm{O}_{3}\right)$ core. $^{37}$ Magnetic nanosheets can be obtained when combining these nanoparticles with freestanding films. LbL assembly allows the achievement of films with large surface area (up to tens of $\mathrm{cm}^{2}$ ) but with controlled thickness at the nanoscale level (on the order of few tens to hundreds of nanometers). ${ }^{38,39}$ Such structures, exhibiting a magnetic component, can be suitable for different applications in the biomedical field. In addition to already known properties as biocompatibility, and flexibility, magnetic structures can be directed toward an injury, positioned, and handled by using noninvasive external tools. In this sense, this work proposes the incorporation of MNPs in CHI-ALG multilayered films constructed using a bottom-up procedure through the alternate deposition of these two polysaccharides (see Scheme 1). We hypothesize that this technology could boost the development of magnetic films with potential for remotely controlled

Scheme 1. (A) Schematic Illustration of the Production Steps of Magnetic-Responsive Membranes ${ }^{a}$; (B) Scheme Showing the Multilayered Rearrangements within the Nanocomposite Membrane
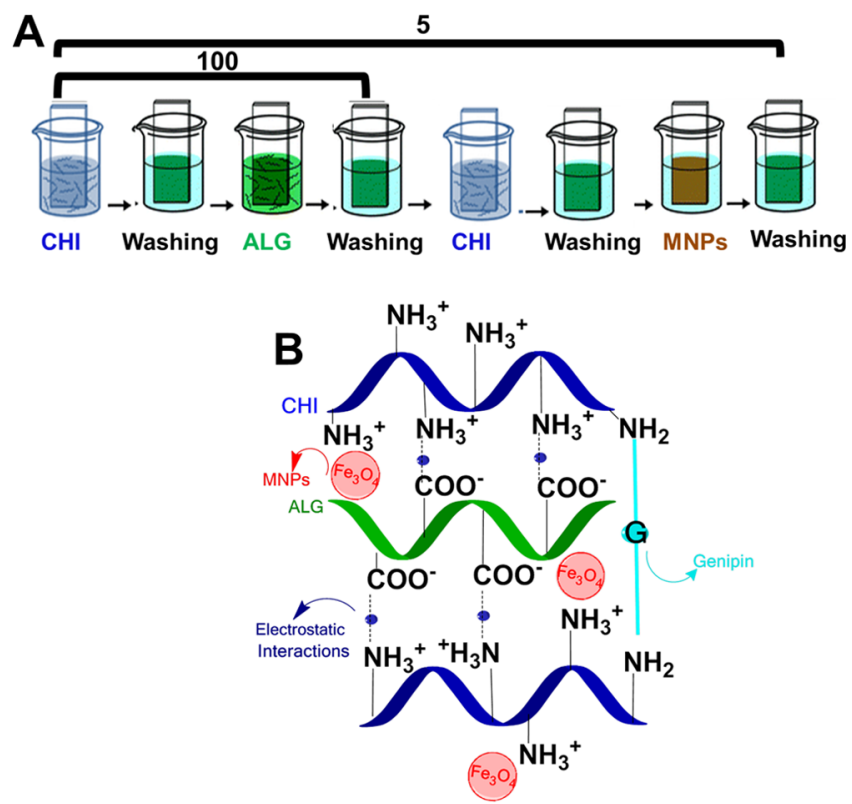

${ }^{a} 100$ bilayers combining $\mathrm{CHI}$ and ALG are first produced, followed by an extra 5 cycles incorporating the MNPs. manipulation by permanent and gradient magnetic fields. Because CHI-ALG freestanding membranes are commonly reported to exhibit low stiffness and poor cell adhesion, a crosslinking step will be added using genipin, a natural product extracted from the gardenia fruit. ${ }^{.4,40,41}$ It is our belief that the additional advantages of magnetic response in highly nanostructured films could be potentially interesting in the development of new devices in biomedicine, in particular in tissue engineering (TE).

\section{MATERIALS AND METHODS}

Synthesis of Magnetic Nanoparticles. Magnetite nanoparticles were synthesized based on the procedure previously published. ${ }^{42}$ Briefly, $\mathrm{Fe}_{3} \mathrm{O}_{4}$ magnetic nanoparticles (MNPs) were synthesized by the coprecipitation reaction of ferrous $\left(\mathrm{FeCl}_{2} \cdot 4 \mathrm{H}_{2} \mathrm{O}\right)$ and ferric $\left(\mathrm{FeCl}_{3} \cdot 6 \mathrm{H}_{2} \mathrm{O}\right)$ salts in the presence of ammonium hydroxide $\left(\mathrm{NH}_{4} \mathrm{OH}\right)$, all from Sigma-Aldrich, USA. The reaction took place under a nitrogen atmosphere at $60^{\circ} \mathrm{C}$. The final product was washed with deionized water and ethanol for several times, and dried overnight in a VD23 (Binder, Germany) vacuum oven. TEM analysis of $\mathrm{Fe}_{3} \mathrm{O}_{4}$ MNPs was observed on a JEM-1010 (JEOL, USA) microscope (100 $\mathrm{kV})$. Samples for TEM were obtained by the evaporation of a drop of the solution sample $\left(1 \mathrm{mg} \mathrm{mL}^{-1}\right)$ on a carbon-coated copper grid, under ambient conditions.

Production of Freestanding Membranes. CHI $\left(M_{\mathrm{w}} 190000-\right.$ 310. $000 \mathrm{Da}, 82.6 \%$ degree of deacetylation, ref 448877, Sigma-Aldrich, USA) and low-viscosity ALG (538 kDa, $\approx 250 \mathrm{cP}$, ref 71238, SigmaAldrich, USA) were the two polyelectrolytes used for the construction of the multilayered films. CHI was purified by a series of filtration and precipitation steps both in water and ethanol. Freestanding membranes were produced using polypropylene supports previously washed in ethanol and water, using a protocol already reported. $24,25,30$ Briefly, polypropylene supports were alternately immersed in $\mathrm{CHI}$ and ALG polyelectrolyte solutions $(0.1 \%(\mathrm{w} / \mathrm{v}), \mathrm{pH} 5.5$, acetate buffer solution $(0.1 \mathrm{M})$ in the presence of additional salt $(0.15 \mathrm{M} \mathrm{NaCl})$ for 8 min each, with an intermediate washing step in acetate buffer solution in between $(4 \mathrm{~min})$. This process was performed using a dipping robot specially designed for the automatic fabrication of multilayers, and was repeated until a 100 bilayer freestanding film of CHI/ALG had been deposited on the templates. In order to fabricate a magnetic responsive system, MNPs were incorporated in both sides of the membranes. After obtaining a 100 bilayer freestanding membrane of CHI-ALG, polypropylene supports were once again alternately immersed in $\mathrm{CHI}$, MNPs $(0.1 \%(\mathrm{w} / \mathrm{v}), \mathrm{pH} 5.5$, acetate buffer solution $(0.1 \mathrm{M})$ in the presence of additional salt $(0.15 \mathrm{M} \mathrm{NaCl}))$ and ALG solutions, followed by an intermediate washing step in an acetate buffer solution (5 cycles), see Scheme 1A. Afterward, membranes were cross-linked with genipin (Wako chemical, USA). Briefly, a genipin solution (3.5 $\mathrm{mg} \cdot \mathrm{mL}^{-1}$ ) was prepared by dissolving the adequate amount of genipin into a dimethyl sulfoxide (Sigma-Aldrich, USA)/sodium acetate buffer (0.15 M NaCl, pH 5.5) mixture (1:4 (v/v)). CHI-ALG and CHI-ALGMNPs membranes were immersed in the cross-linking agent solution and incubated at $37{ }^{\circ} \mathrm{C}$ overnight. Afterward, membranes were thoroughly washed with ethanol to dissolve the amount of unreacted genipin, and left to dry at room temperature. CHI-ALG and CHIALG-MNPs multilayered films without cross-linking were used as controls.

Membranes Morphology and Composition. The surface of CHI-ALG membranes with or without cross-linking, and with or without incorporated MNPs was visualized by scanning electron microscopy (SEM). Membranes were gold-sputtered and visualized using a Jeol JSM-6010LV microscope operating at an accelerating voltage of $15 \mathrm{kV}$. In addition, energy dispersive spectroscopy (EDS) mapping was also performed. All samples were fixed by mutual conductive adhesive tape. Samples were analyzed in order to visualize the appearance of an iron component in the CHI/ALG membranes with or without cross-linking and with incorporated MNPs. Membranes without incorporated MNPs were used as control. 
Shape Memory. All the formulations of freestanding membranes obtained by the LbL technique exhibited an initial flat permanent shape that upon hydration could be elastically deformed into an helicoidally shape. Afterward, the shape of the deformed membrane was fixed by dehydration with ethanol $100 \%$ in order to accelerate the extraction of water molecules. This stable shape (temporary shape) was retained until the membrane was hydrated again in distilled water. Additionally, the cross-linking reaction with genipin was also used to surpass the limitation imposed by the initial flat geometry of the fabricated membranes, permitting to obtain permanent shapes with other geometrical configurations.

Water Uptake. The water-uptake ability of the freestanding membranes was measured by soaking dry freestanding membranes of known weight in phosphate buffer (PBS, Sigma, USA) at $37^{\circ} \mathrm{C}$. The swollen membranes were removed after $12 \mathrm{~h}$. After removing the excess of PBS using a filter paper (Filter Lab, Spain), the freestanding membranes were weighed with an analytical balance (Denver Instrument, Germany). The water uptake was calculated as follows

$$
\text { water uptake } \%=\frac{W_{\mathrm{w}}-W_{\mathrm{d}}}{W_{\mathrm{d}}} 100
$$

where $W_{\mathrm{w}}$ and $W_{\mathrm{d}}$ are the weights of swollen and dried freestanding membranes, respectively.

Mechanical Tests. Mechanical properties of freestanding membranes were studied in the wet state using an INSTRON 5540 (INSTRON Int., Ltd., High Wycombe, UK) universal testing machine with a cell load of $1 \mathrm{kN}$. Data presented is a result of at least three independent measurements. The dimensions of the specimens used were $60 \mathrm{~mm}$ in length, $6.5 \mathrm{~mm}$ in width, and $38 \pm 2.5,41.2 \pm 3.4,34$ \pm 3.7 , and $40.3 \pm 4.5 \mu \mathrm{m}$ in thickness for CHI/ALG membranes with or without cross-linking, and with or without incorporated MNPs, respectively. The load was placed midway between the supports with a span of $10 \mathrm{~mm}$. The crosshead speed was $1 \mathrm{~mm} \mathrm{~min}{ }^{-1}$. For each condition, the specimens were loaded until core break. Five samples were used per condition.

Cell Culture. To evaluate the in vitro biological performance of all the freestanding membrane's formulation, cell culture studies were performed with L929, a mouse fibroblast of connective tissue cell line (European Collection of Cell Cultures (ECCC, UK). Cells were cultured in Dulbecco's modified Eagle's medium (DMEM; Sigma, USA), supplemented by $10 \%$ heat-inactivated fetal bovine serum (FBS; Biochrom AG, Germany) and 1\% antibiotic- antimicotic (Gibco, USA). Prior to cell seeding (25000 cells per well) membranes were sterilized with $70 \%(\mathrm{v} / \mathrm{v})$ ethanol overnight and rinsed three times in PBS.

Morphological Characterization: DAPI-Phalloidin and Scanning Electron Microscopy (SEM). The different formulations of freestanding membranes were incubated for 1,3 , and 7 days at $37^{\circ} \mathrm{C}$ in a humidified 5\% $\mathrm{CO}_{2}$ atmosphere. 4,6-Diaminidino-2-phenylindoledilactate (DAPI, $20 \mathrm{mg} \mathrm{mL}^{-1}$, Sigma-Aldrich, USA) and phalloidintetramethylrhodamine $\mathrm{B}$ isothiocyanate dyes (phalloidin, $10 \mathrm{mg} \mathrm{mL}^{-1}$, Sigma-Aldrich, USA) were used to perform a DAPI-phalloidin assay ( $n=3$ samples per well, in triplicate). Briefly at each time point, culture medium was removed and the samples fixed in $10 \%$ formalin. After $1 \mathrm{~h}$, formalin was removed and replaced by PBS. Upon PBS washing, $1 \mathrm{~mL}$ of PBS containing $5 \mu \mathrm{L}$ of phalloidin was added for 40 $\mathrm{min}$ at room temperature and protected from light. After extensively washing with PBS, samples were stained with DAPI by adding $1 \mu \mathrm{L}$ of it in $1 \mathrm{~mL}$ of PBS for $10 \mathrm{~min}$. After DAPI staining, samples were washed three times with PBS and visualized in the dark by inverted fluorescent microscope (Zeiss, Germany). For SEM analysis samples were dehydrated, sputtered-coated with gold, and visualized by SEM (Jeol, USA).

Cellular Viability Assay. The viability of cells seeded on freestanding membranes was also tested using a MTS colorimetric assay (Cell Titer 96AQueous One Solution Cell Proliferation Assay, Promega, USA) at 1, 3, and 7 days of culture. Briefly, samples were washed with PBS and immersed in a mixture consisting of serum-free cell culture medium and MTS reagent in a 5:1 ratio and incubated for
$3 \mathrm{~h}$ at $37^{\circ} \mathrm{C}$ in a humidified atmosphere containing $5 \% \mathrm{CO}_{2}$. After this, $100 \mu \mathrm{L}$ of each well (in triplicate) were transferred to a 96-well plate. The amount of formazan product was measured by absorbance at a wavelength of $490 \mathrm{~nm}$ using a microplate spectrophotometer (Synergy HT, Bio-TEK, USA). The background was corrected by subtracting the absorbance obtained from freestanding membranes without cells to those with cells.

Statistical Analysis. Statistical analysis was performed with Graphpad Prism version 5.0 for Windows (Graphpad software, USA). All variables are expressed as mean \pm standard deviation (SD) from at least three independent experiments. Nonparametric (Kruskal-Wallis test) or parametric tests (one way ANOVA followed by Turkey test) depending on whether the samples were from normally distributed populations or not, respectively.

\section{RESULTS AND DISCUSSION}

Morphology and Composition. CHI and ALG, or CHI, MNPs, and ALG, were sequentially adsorbed on the surface of hydrophobic polypropylene substrates to produce CHI/ALG or CHI-ALG-MNPs membranes. Freestanding membranes were easily detached from the polypropylene substrate due to weak van der Waals forces between the initial CHI layer and the substrate. With 100 bilayers, membranes were found to be robust, compliant, and easy to handle which allowed the shaping of the membranes in virtually any shape. ${ }^{24,25,30}$ Additionally, freestanding films allowed the direct experimental determination of many physicochemical and mechanical properties of fundamental significance without the influence of the substrate, which is just used as a processing platform.

All the formulations of freestanding membranes were first assessed at the naked eye (Figure 1). Photographs reveal an

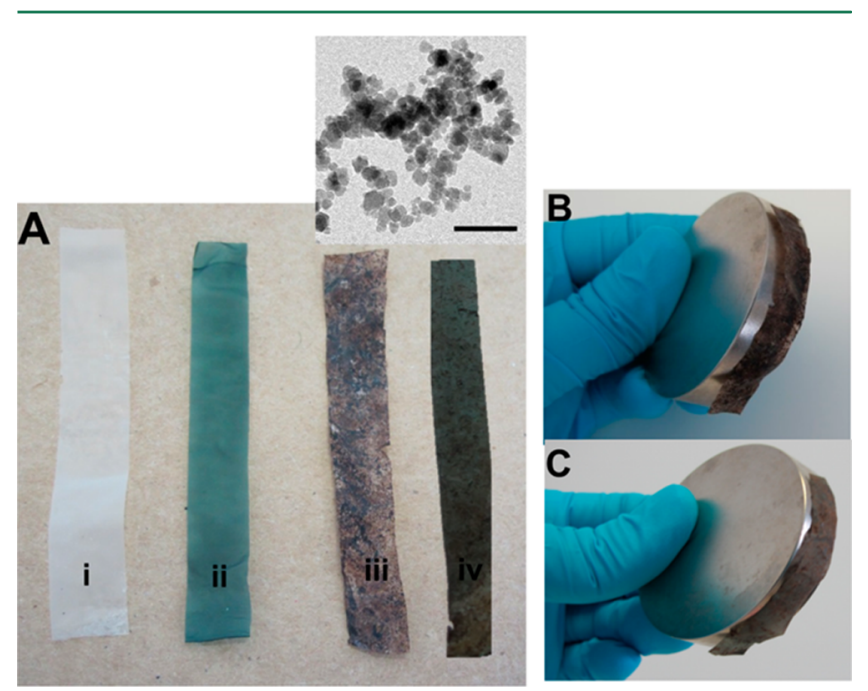

Figure 1. (A) Photographs of all membrane formulations: (i) CHIALG, (ii) CHI-ALG with genipin (G) cross-linking, (iii) CHI-ALGMNPs, (iv) CHI-ALG-G-MNPs. The inset image represent TEM micrographs of MNPs. The scale bar is $50 \mathrm{~nm}$. Magnetic response of freestanding membranes upon application of an external magnetic field for (B) CHI-ALG-MNPS and (C) CHI-ALG-G-MNPS freestanding membranes.

homogeneous morphology in all the formulations but different colors due to the chemical cross-linking and/or inclusion of MNPs. Genipin cross-linking has the ability to cross-link polymers containing amine-groups, generating a greenish color on the samples, as previously reported. This effect has been explained by oxygen-radical induced polymerization of genipin, as well as with its reaction with amine groups. ${ }^{43,44}$ However, the 


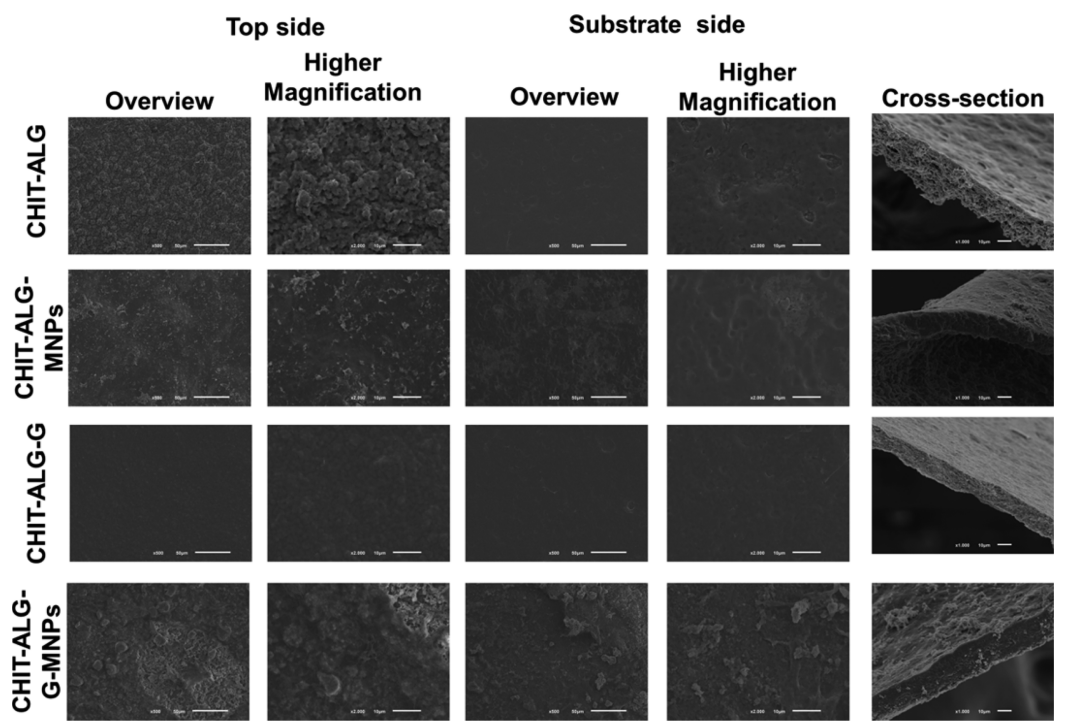

Figure 2. Morphology of the produced membranes: SEM micrographs of the top side and substrate side of the membranes. The scale bars are 50 and $10 \mu \mathrm{m}$ for the overview and higher magnification, respectively. Cross-section micrographs of the different freestanding membranes were also performed. The scale bar is $10 \mu \mathrm{m}$.

main aim of this work was not to study the genipin crosslinking mechanism but evaluate the magneto-active propensity of these polysaccharide membranes.

Membranes with incorporated MNPs presented a dark brown appearance due to the magnetite content of the freestanding membranes. Additionally, all the membranes presented a dark brown color which indicates a homogeneous dispersion of MNPs and a good interfacial bonding and interaction between the MNPs and the PEMs. MNPs were incorporated within the multilayers by electrostatic interactions because of their negative charge $(-37.9 \pm 0.3 \mathrm{mV})^{42}$ and also by van der Waals interactions, which results in an apparent uniform film with inorganic filler well-dispersed, and integrated in polyelectrolyte multilayers (PEMs). However, considering that the MNPs might diffuse across the film (which was not detected during the manipulation of the membranes, including during cell culture) genipin cross-linking was used to improve the stability of the multilayers decrease the void space between the layers, which in turn led to a lower diffusion across the membrane. Similar protocols were also used in LbL composite multilayers based on silica nanoparticles and polycyclic aromatic hydrocarbons (PAH) as the components. ${ }^{45}$

Figure 1B, C as well as Videos S1-S4 show the response of freestanding membranes upon application of an external magnetic field. As expected, the obtained freestanding membranes retain the magnetic properties of the nanoparticles. The magnetic flux density generated by the external magnet attracts the CHI-ALG-MNPs (with cross-linking or without cross-linking) membranes. The same effect cannot be replicated if the freestanding do not possess incorporated MNPs.

The proposed technique allows the production of flexible freestanding membranes which can be manipulated and precisely positioned within the working environment by using an external (stimuli magnetic field) and could thus provide a novel controllable support in biomedical applications. Traditional multilayered membranes were proposed as an alternative for wound sutures, patches, bone defect covers, or as flexible cell growth supports. The responsiveness of theses membranes to magnetic fields will allow to finely manipulate the membranes within wet or liquid environments by using noninvasive external tools. The use of incorporated magnetic nanoparticles in freestanding membranes may function as gastrointestinal patches, ${ }^{33}$ contrast agents for magnetic resonance imaging, ${ }^{46} \mathrm{TE}$ approaches for bone repair (bone implant fixation, local drug delivery, mimicking microenvironment for stem cell differentiation, ${ }^{47}$ hyperthermia-based therapy $^{48}$ and controlled drug delivery of growth factors ${ }^{49}$ and/or bioactive molecules for stented blood vessels. ${ }^{50}$

The morphology of the developed freestanding membranes was evaluated by SEM (Figure 2). The results reveal an homogeneous morphology of all the membranes and, consequently, an uniform deposition of multilayers. The absence of defects reveals the efficacy of the detachment of the multilayers using these supports. The SEM analysis was made using the upper part of the membrane (ALG side) and the substrate side (CHI side). As expected and previously reported, the substrate side (side in contact with the substrate prior to the detachment) is smoother. ${ }^{24}$

The results reveal a similar morphology between the samples. However, the cross-linked membranes present a smoother surface which corroborated previous results obtained in CHIALG membranes. ${ }^{14,24,25,30}$ With the addition of MNPs, an increase in roughness occurs, which indicates their effective inclusion into the multilayers. The presence of MNPs was further confirmed by EDS: membranes with incorporated MNPs presented Fe content, that was not detected in the control membranes (Figure 3).

The thickness of the freestanding membranes was also determined using cross sections of all the formulations used (Figure 2). CHI-ALG membranes presented a dry thickness of $19.3 \pm 4.4$ or $17.5 \pm 2.9 \mu \mathrm{m}$ without and with MNPs, respectively. On the other side, a dry thickness of $21.2 \pm 2.5$ or $23 \pm 3.04 \mu \mathrm{m}$ was attained for cross-linked membranes without or with MNPs, respectively. These results are in accordance with earlier results, where cross-linking increased the stiffness of the multilayers without significant changes in PEMs' thickness in the dry state. ${ }^{30}$ Additionally, the cross-sections of all the freestanding membranes also revealed some porosity. For biomedical applications, such porosity could be beneficial, for example, to enhance nutrient transport to cells or to increase 


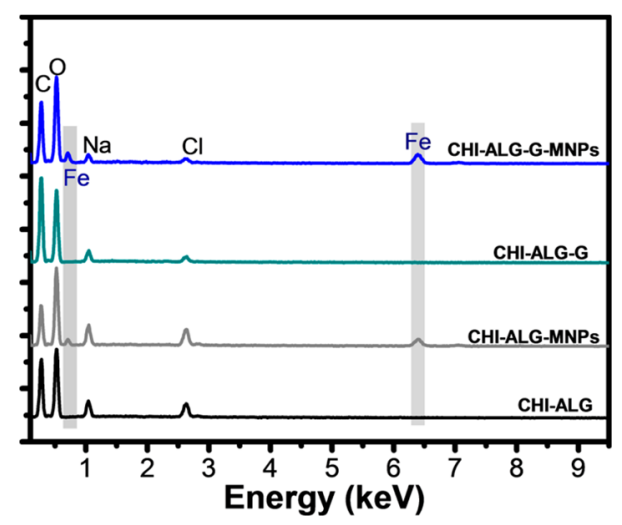

Figure 3. EDS spectra of the produced membranes formulations.

the surface area. The diffusion properties of CHI/ALG membranes were already reported in previous studies. $24,26,30$

Shape Memory. The shape memory effect offers novel materials-based devices that can surpass some of today's scientific challenges, for solving scientific challenges due to their demonstrated ability to actively undergo geometric transformation upon exposure to environmental stimuli, such as hydration. ${ }^{51}$ The shape memory capability of freestanding membranes with and without MNPs was evaluated. Figure 4 shows representative photographs of water-triggered shape memory properties of control membranes (CHI-ALG, CHIALG-G) and membranes with incorporated MNPs.

In all the cases, membranes lifted off from the substrate presented a flat permanent shape that upon hydration can be virtually deformed in any shape (in this case, an helicoidal shape). After elastical deformation, the helicoidal shape was fixed by dehydration which led to a stable temporary shape. The dehydration step was performed with pure ethanol, allowing a fast extraction of plasticizing water molecules. The permanent shape of the membrane can be easily recovered by hydration, being the process completely reversible (see Video S5). This process has been previously explored and it is intrinsically related with the molecular mobility and glass transition dynamics of PEMs. ${ }^{52}$ The glass transition of the freestanding membranes is highly dependent on the water content, and in this sense, a minimum hydration level to promote the shape memory recovery is strictly necessary.

Additionally the use of genipin as cross-linking offered the possibility to surpass the limitation imposed by the permanent shape, determined by the geometry of the processed membrane, allowing us to have several permanent shapes. Such shapes could be easily adapted for several applications, such as the biomedical ones. Using this methodology, freestanding membranes with and without MNPs were deformed into an helicoidal temporary shape by hydration, deformation and winding around a mandrel-see Figure 5. This temporary shape was fixed into a new and stable permanent shape using in this case genipin cross-linking. The dry crosslinked membranes became rigid and could not deform freely, keeping the helicoidal shape. The cross-linked membranes were further deformed by hydration and its geometry was fixed by dehydration, leading to a new flat temporary shape. Upon rehydration, the membranes became soft and easily deformed, recovering their initial helicoidal shape (Figure 5).

These kinds of materials are ideal candidates for biomedical applications in which the temporary shape has to be preserved until the device is inserted in the defect/cavity to be filled, and

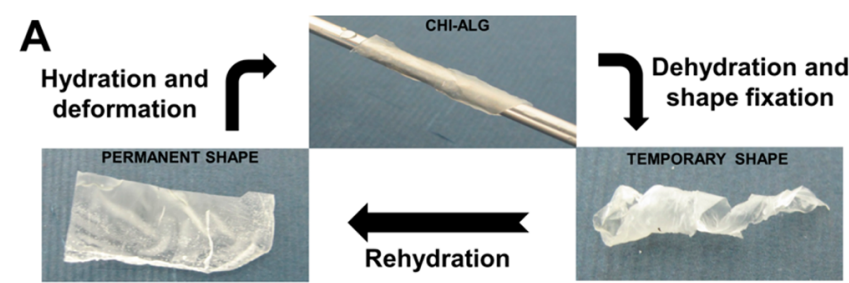

B

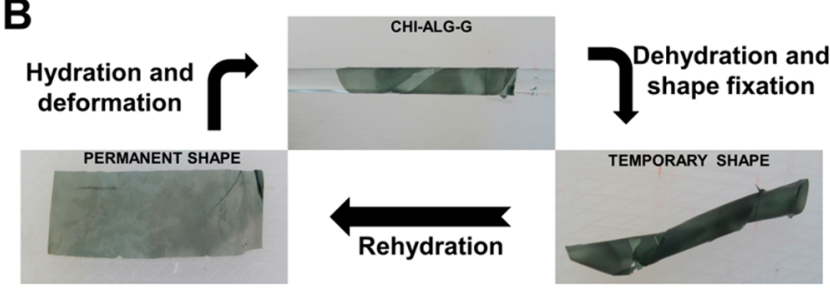

C

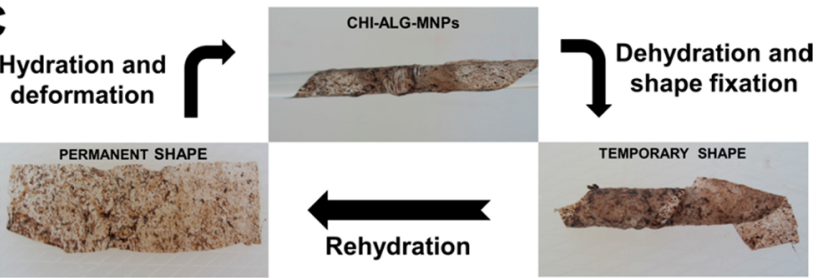

D

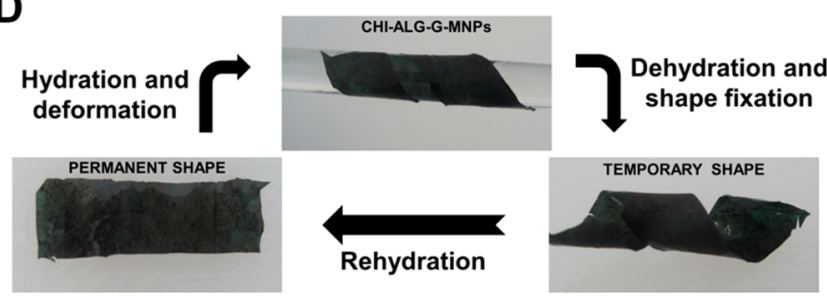

Figure 4. Optical photographs that demonstrate the hydration triggered shape memory of native (A) CHIT-ALG and genipin cross-linked membranes (B) CHI-ALG-G, (C) CHI-ALG-MNPs, and (D) CHI-ALG-G-MNPs.

A

PERMANENT SHAPE

TEMPORARY SHAPE
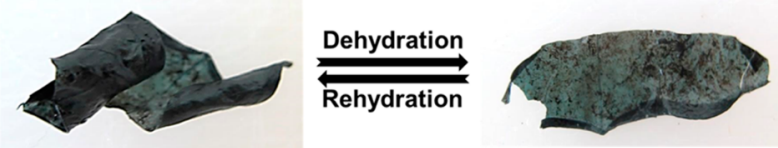

B
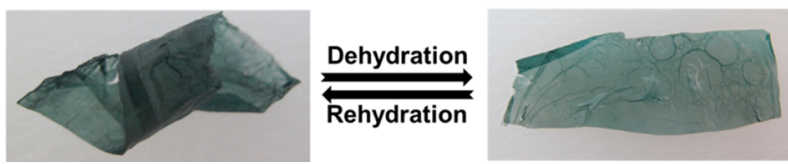

Figure 5. Optical photographs that demonstrate the possibility of multilayer membranes with nonflat permanent shapes, exhibiting shape memory triggered by hydration: (A) CHI-ALG-G-MNPS and (B) CHI-ALG-G.

then reach their application, i.e., their permanent shape when a certain hydration level is achieved. The additional magnetic responsiveness of the nanocomposite membrane could allow an extra capability for the device to adapt and fix to the implanted site.

Swelling Properties. Membranes based on CHI and ALG are inherently composed by a considerable number of hydrophilic groups such as amine, carboxyl and hydroxyl groups which can promote the water uptake. ${ }^{53,54}$ The swelling ability of the prepared membranes was evaluated in phosphate 

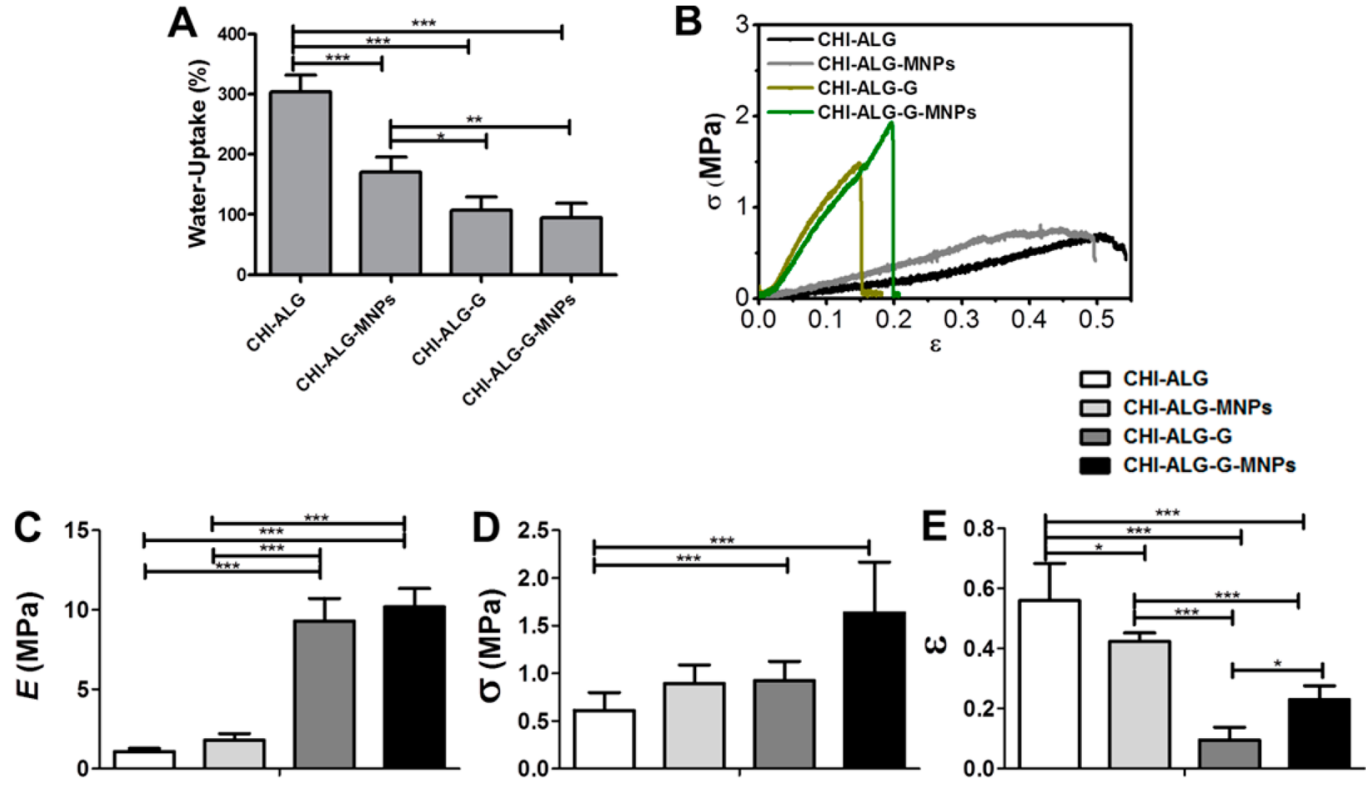

Figure 6. Physicochemical characterization of the developed freestanding membranes formulations. (A) Swelling ability of native and cross-linked membranes with and without MNPs (PBS, $37^{\circ} \mathrm{C}$ ). (B) Strain-stress curves for all the freestanding membrane's formulations, (C) Young modulus $(E),(D)$ ultimate tensile strain $(\sigma)$, and $(E)$ maximum extension $(\varepsilon)$. Significant differences were found for $p<0.05(*)$ and $p<0.001(* * *)$.
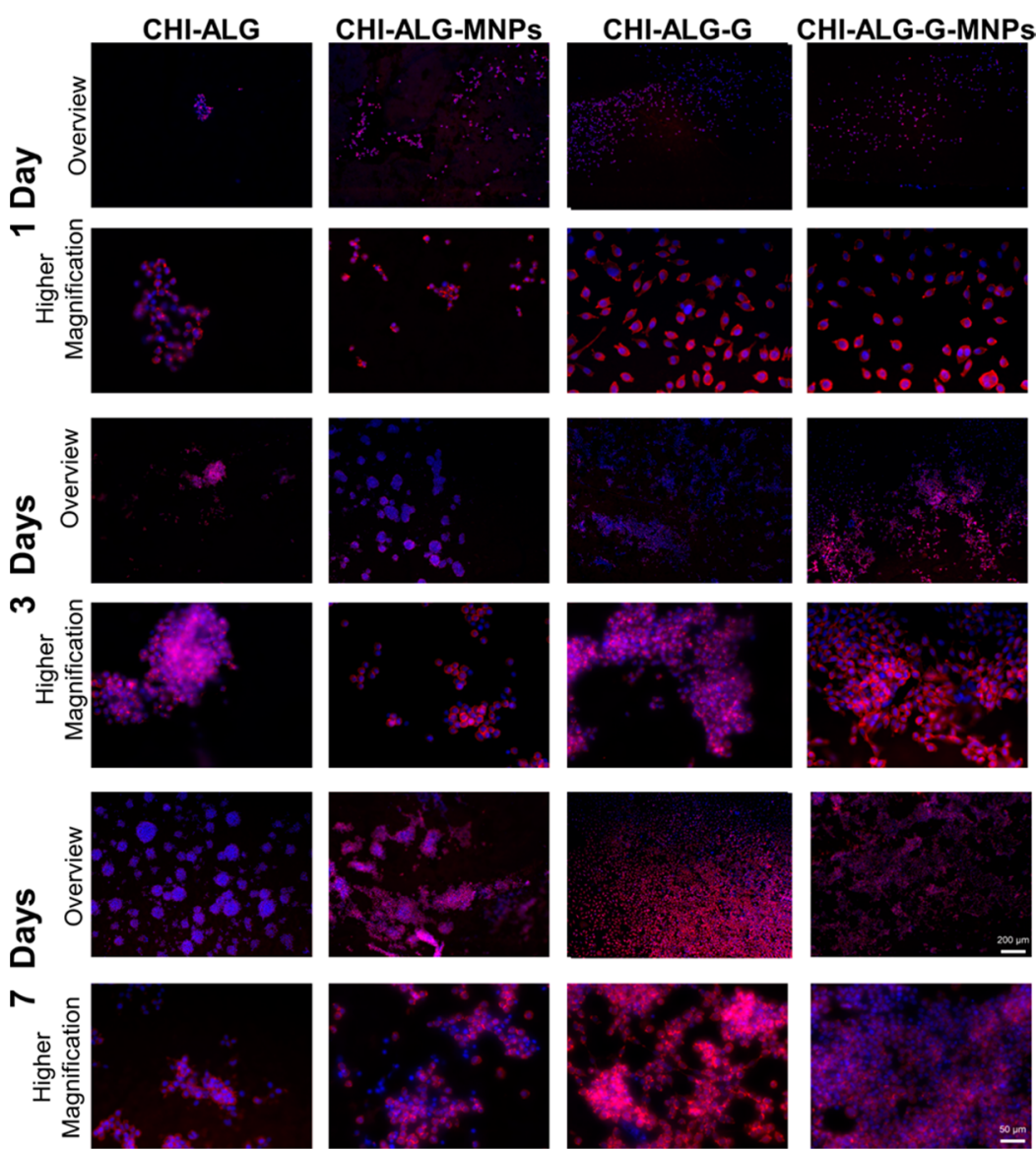

Figure 7. DAPI-phalloidin fluorescence assay at 1,3, and 7 days of culture in all the membranes formulations. Cells nuclei were stained blye by DAPI and F-actin filaments in red by phalloidin. Scale bars represents 200 and $50 \mu \mathrm{m}$ in lower- and higher-magnification images, respectively.

buffer at $37{ }^{\circ} \mathrm{C}$ overnight (Figure $6 \mathrm{~A}$ ). The chemical crosslinking with genipin led to a higher reduction on water-uptake due to the smaller free volume and intermolecular space between the polyelectrolyte chains, which limits the molecular mobility of the polymer chains at the nanoscale level, as previously reported. $^{25}$ The incorporation of MNPs on native freestanding membranes also led to a reduction on the wateruptake ability because these nanoparticles are not able to 


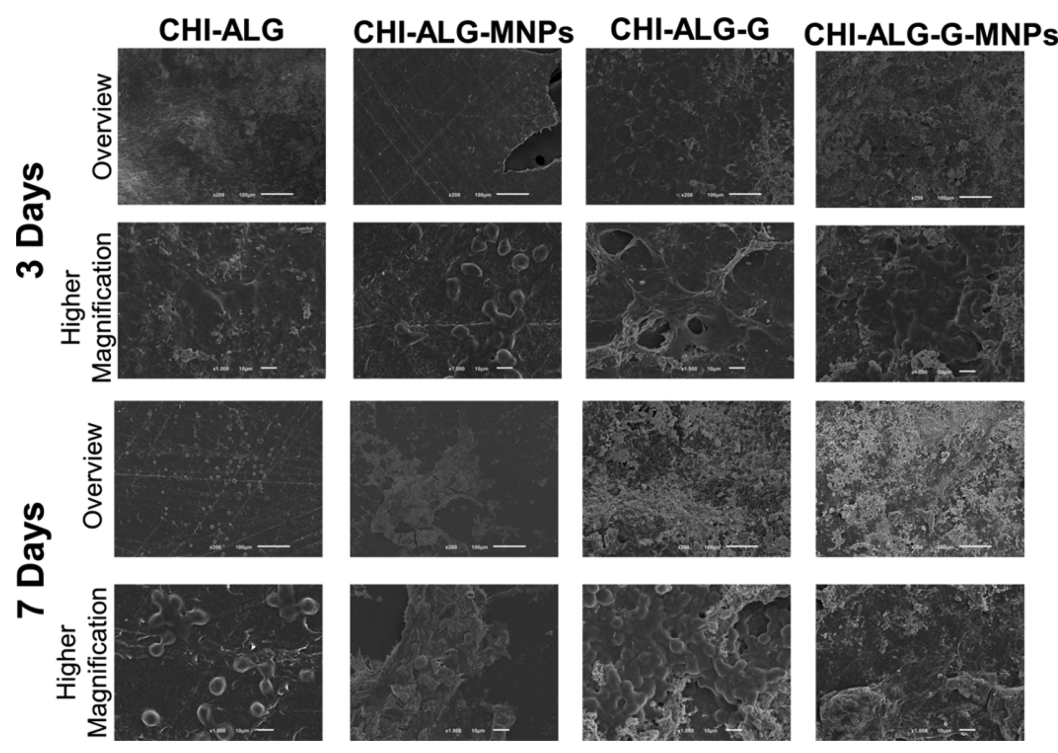

Figure 8. SEM images of L929 seeded on all the freestanding formulations at day 3 and day 7 of culture. Scale bar represents 50 and $10 \mu \mathrm{m}$ in lowerand higher-magnification images, respectively.

adsorb water. The decreasing of swelling for CHI-ALG-G freestanding membranes containing MNPs was lower when compared with the ones without cross-linking. This can be explained by the fact that the number of layers containing MNPs is much lower (i.e., 10 bilayers) than the ones containing CHIT-ALG (i.e., 100 bilayers). Thus, the cross-linking effect prevails when compared with the incorporation of MNPs.

Mechanical Properties. The mechanical properties of freestanding membranes were evaluated in the wet state using an Universal Mechanical Testing machine. Figure 6B shows representative stress-strain curves for all the formulations. The results show a tendency for an increase of Young modulus $(E)$, ultimate tensile strength $(\sigma)$ with the genipin cross-linking, as well as with the incorporation of MNPs (Figure 6C, D). However, the maximum extension in both cases decrease, since the structures became more brittle (Figure 6E). The same behavior has been previously reported when CHI-ALG freestanding membranes were cross-linked with genipin. ${ }^{25,30}$

With the presence of MNPs as second component of the multiphase nanocomposite, the mechanical properties increased an effect that can be explained by classic composite models, such as Takayanagi model. ${ }^{55}$ According to this model, the modulus of a composite material can be calculated as the composite modulus of the components by taking in consideration their volume fraction and the composite morphology of the membranes. The same behavior has been previously reported for gold ${ }^{13}$ and magnetite nanoparticles ${ }^{35}$ when included in multilayered systems.

All together, these results confirm the hypothesis that crosslinking of PEMs with genipin substantially increases the mechanical properties of the freestanding membranes when compared with the inclusion of MNPs.

Biological Performance. The potential of CHI-ALG freestanding membranes with cross-linking and/or with MNPs incorporated was evaluated in vitro with L929 cells. Cell adhesion and morphology was studied by DAPI-phalloidin assay and also by SEM observation (Figures 7 and 8). Both results were concordant. Cells seeded on the membranes adhere on the surface but behave quite different between all the formulations used. As expected and previously reported the cross-linking has a positive effect on specific cellular events, because it is well-known to decrease its water content, increasing the stiffness. ${ }^{14}$ The cells seeded on cross-linked membranes are well spread and anchored to the film, presenting a stretched morphology. On the other side, the cells cultured on native membranes are less susceptible to creating strong anchors, which can occur because of the softness and, especially, high hydration of these films. Thus, the cells seeded on these films tend to aggregate, presenting a round morphology.

With these results, it is also possible to verify an increase in cell number with increasing culture time. However, in crosslinked membranes without and with MNPs, cells proliferate in dense layers, whereas on native membranes, they are organized in clusters. The same trend was also verified for CHI-ALG membranes containing MNPs besides the higher mechanical properties and lower hydration. Clusters have been widely reported as prominent in cases of deficient cell-matrix interactions, which protect them against anoikis, that is, apoptosis induced by poor adhesion. ${ }^{41}$

Regarding the cell viability assessment, in general the results revealed that cells were able to remain viable in the membranes up to 7 days in culture (Figure 9). Additionally, the MTS

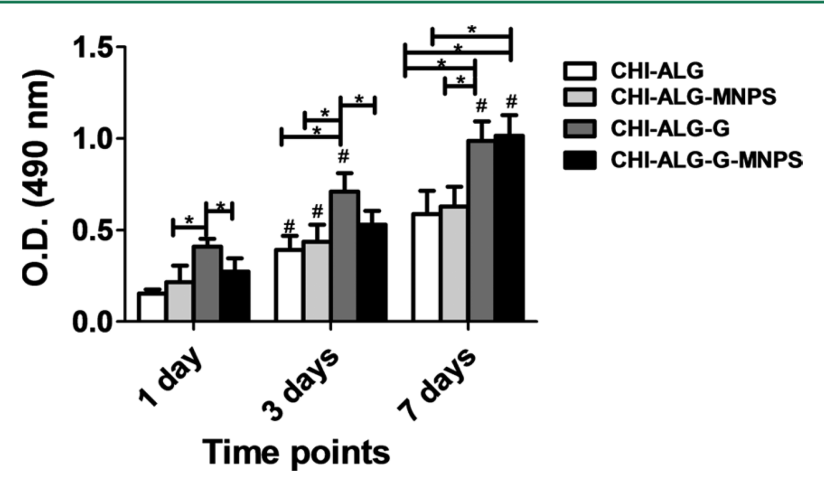

Figure 9. Cell viability at 1,3 , and 7 days of culture in the different freestanding membrane formulations by MTS assay. Significant differences were found for $p<0.05(*)$. 
results corroborated the results obtained in DAPI-phalloidin assay and SEM because a statistically significant enhancement was observed with the inclusion of MNPs as well as with genipin cross-linking. These results are a consequence of better cell adherence and spreading in the formulation with higher mechanical properties and lower hydration levels. This behavior is consistent with other studies reported in literature for other types of cells (fibroblasts, smooth muscle cells, and neurons) using different PEMs. ${ }^{56-58}$ Tailoring the mechanical properties of PEMs play an important role in many cellular processes ranging from motility, to spreading, proliferation and differentiation. ${ }^{57,59,60}$ Gathering all the results, this work demonstrates the important role of membrane mechanics (crosslinking or incorporation of fillers) in cell adhesive and proliferation properties.

\section{CONCLUSIONS}

In summary we have demonstrated the successful and facile production of functional nanocomposite multilayered freestanding membranes by simple control of the coupling between the multilayered films and the MNPs. Using polypropylene supports as template, it was possible to obtain a robust, compliant and flexible freestanding membranes with magnetic properties whose architecture can have an unprecedented control at the nanometer and micrometer scale. The results showed that MNPs have a minor effect in the morphology of freestanding membranes. The magnetic character of freestanding membranes with intercalated layers of MNPs was confirmed by EDS analysis, which revealed the presence of iron $(\mathrm{Fe})$ in the membranes. In addition, the application of a remotely and controllable external magnetic field also confirmed their magnetic field responsiveness. The mechanical properties were also evaluated, and it was observed that the presence of MNPs increases the Young modulus and ultimate tensile strength of the multilayered membranes. The shape memory property of the freestanding membranes triggered by hydration was also confirmed, and we consider that it constitutes a promising feature for their use as smart materials in a plethora of research fields, especially in biomedical fields e.g. implantable devices where the $3 \mathrm{D}$ devices should be implanted in empty and hydrate regions, such as in bone and cartilage defects. The in vitro biological performance demonstrates that the inclusion of MNPs affected and enhanced cell behavior regarding their viability, adhesion, and morphology. Our results suggest that the developed magneticresponsive freestanding membranes obtained mainly by LbL methodology could open new perspectives in TE approaches and in flexible biomedical devices for drug delivery field. Other functionalities, and properties associated with the magnetic component of freestanding membranes will be envisaged in future studies, namely their incorporation into three-dimensional tissues. As foreseen in previous studies, magnetic films can be manipulated and precisely positioned by using an external magnetic field, and could offer a new controllable support in TE. ${ }^{50}$ Classical examples of biomedical applications of flexible freestanding membranes would be their use as gastrointestinal patches, ${ }^{33}$ contrast agents for magnetic resonance imaging, ${ }^{46} \mathrm{TE}$ approaches for bone repair (bone implant fixation, local drug delivery, and mimicking microenvironment of stem cell differentiation), ${ }^{47}$ hyperthermia-based therapy, ${ }^{48}$ and controlled drug delivery of growth factors ${ }^{49}$ and/ or bioactive molecules for stented blood vessels. ${ }^{50}$ The responsiveness of theses membranes to magnetic fields, together with the ability to easily tune different properties in one structure, are the most promising features of the proposed system toward the development of flexible biodevices with potential use in TE.

\section{ASSOCIATED CONTENT}

S Supporting Information

The Supporting Information is available free of charge on the ACS Publications website at DOI: 10.1021/acsbiomaterials.5b00292.

Freestanding membranes without MNPs, Video S1 (AVI)

Freestanding membranes with MNPs, Video S2 (AVI)

Freestanding membranes with cross-linking and without MNPs, Video S3 (AVI)

Freestanding membranes with cross-linking and MNPs, Video S4 (AVI)

Shape memory of freestanding membranes based on CHI-ALG-MNPs, Video S5 (AVI)

\section{AUTHOR INFORMATION}

\section{Corresponding Author}

*E-mail: jmano@dep.uminho.pt.

\section{Author Contributions}

${ }^{\dagger}$ S.G. and J.M.S. contributed equally to this work.

\section{Notes}

The authors declare no competing financial interest.

\section{ACKNOWLEDGMENTS}

The authors acknowledge the financial support by the Portuguese Foundation for Science and Technology (FCT) through the Doctoral grants with the reference numbers SFRH/BD/81372/2011 (JMS). This work was financially supported by the FCT, by the project PTDC/FIS/115048/ 2009.

\section{REFERENCES}

(1) Tsukruk, V. V. Assembly of supramolecular polymers in ultrathin films. Prog. Polym. Sci. 1997, 22 (2), 247-311.

(2) Luzinov, I.; Minko, S.; Tsukruk, V. V. Adaptive and responsive surfaces through controlled reorganization of interfacial polymer layers. Prog. Polym. Sci. 2004, 29 (7), 635-698.

(3) Kang, T. J.; Cha, M.; Jang, E. Y.; Shin, J.; Im, H. U.; Kim, Y.; Lee, J.; Kim, Y. H. Ultra-thin and Conductive Nanomembrane Arrays for Nanomechanical Transducers. Adv. Mater. 2008, 20 (16), 3131-3137.

(4) Zeng, T.; Claus, R.; Zhang, F.; Du, W.; Cooper, K. L. Ultrathin film actuators fabricated by layer-by-layer molecular self-assembly. Smart Mater. Struct. 2001, 10 (4), 780.

(5) Yang, M.; Yang, Y.; Yang, H.; Shen, G.; Yu, R. Layer-by-layer selfassembled multilayer films of carbon nanotubes and platinum nanoparticles with polyelectrolyte for the fabrication of biosensors. Biomaterials 2006, 27 (2), 246-255.

(6) Tang, Z.; Wang, Y.; Podsiadlo, P.; Kotov, N. A. Biomedical Applications of Layer-by-Layer Assembly: From Biomimetics to Tissue Engineering. Adv. Mater. 2006, 18 (24), 3203-3224.

(7) Zhai, L.; Nolte, A. J.; Cohen, R. E.; Rubner, M. F. pH-Gated Porosity Transitions of Polyelectrolyte Multilayers in Confined Geometries and Their Application as Tunable Bragg Reflectors. Macromolecules 2004, 37 (16), 6113-6123.

(8) Jaber, J. A.; Schlenoff, J. B. Recent developments in the properties and applications of polyelectrolyte multilayers. Curr. Opin. Colloid Interface Sci. 2006, 11 (6), 324-329. 
(9) Boudou, T.; Crouzier, T.; Ren, K.; Blin, G.; Picart, C. Multiple Functionalities of Polyelectrolyte Multilayer Films: New Biomedical Applications. Adv. Mater. 2010, 22 (4), 441-467.

(10) de Villiers, M. M.; Otto, D. P.; Strydom, S. J.; Lvov, Y. M. Introduction to nanocoatings produced by layer-by-layer (LbL) selfassembly. Adv. Drug Delivery Rev. 2011, 63 (9), 701-715.

(11) Detzel, C.; Larkin, A.; Rajagopalan, P. Polyelectrolyte Multilayers in Tissue Engineering. Tissue Eng., Part B 2011, 17 (2), $101-113$.

(12) Lvov, Y.; Ariga, K.; Ichinose, I.; Kunitake, T. Formation of Ultrathin Multilayer and Hydrated Gel from Montmorillonite and Linear Polycations. Langmuir 1996, 12 (12), 3038-3044.

(13) Jiang, C.; Markutsya, S.; Tsukruk, V. V. Compliant, Robust, and Truly Nanoscale Free-Standing Multilayer Films Fabricated Using Spin-Assisted Layer-by-Layer Assembly. Adv. Mater. 2004, 16 (2), 157-161.

(14) Hillberg, A. L.; Holmes, C. A.; Tabrizian, M. Effect of genipin cross-linking on the cellular adhesion properties of layer-by-layer assembled polyelectrolyte films. Biomaterials 2009, 30 (27), 44634470.

(15) Alves, N. M.; Picart, C.; Mano, J. F. Self assembling and crosslinking of polyelectrolyte multilayer films of chitosan and alginate studied by QCM and IR spectroscopy. Macromol. Biosci. 2009, 9 (8), $776-785$.

(16) Martins, G. V.; Mano, J. F.; Alves, N. M. Dual responsive nanostructured surfaces for biomedical applications. Langmuir 2011, 27 (13), 8415-8423.

(17) Ariga, K.; Hill, J. P.; Ji, Q. Layer-by-layer assembly as a versatile bottom-up nanofabrication technique for exploratory research and realistic application. Phys. Chem. Chem. Phys. 2007, 9 (19), 23192340.

(18) Hammond, P. T. Engineering materials layer-by-layer: Challenges and opportunities in multilayer assembly. AIChE J. 2011, 57 (11), 2928-2940.

(19) Matsusaki, M.; Ajiro, H.; Kida, T.; Serizawa, T.; Akashi, M. Layer-by-Layer Assembly Through Weak Interactions and Their Biomedical Applications. Adv. Mater. 2012, 24 (4), 454-474.

(20) Costa, R. R.; Mano, J. F. Polyelectrolyte multilayered assemblies in biomedical technologies. Chem. Soc. Rev. 2014, 43 (10), 34533479.

(21) Correia, C. R.; Reis, R. L.; Mano, J. F. Multilayered Hierarchical Capsules Providing Cell Adhesion Sites. Biomacromolecules 2013, 14 (3), 743-751.

(22) Szarpak, A.; Cui, D.; Dubreuil, F.; De Geest, B. G.; De Cock, L. J.; Picart, C.; Auzély-Velty, R. Designing Hyaluronic Acid-Based Layerby-Layer Capsules as a Carrier for Intracellular Drug Delivery. Biomacromolecules 2010, 11 (3), 713-720.

(23) Larkin, A. L.; Davis, R. M.; Rajagopalan, P. Biocompatible, Detachable, and Free-Standing Polyelectrolyte Multilayer Films. Biomacromolecules 2010, 11 (10), 2788-2796.

(24) Caridade, S. G.; Monge, C.; Gilde, F.; Boudou, T.; Mano, J. F.; Picart, C. Free-Standing Polyelectrolyte Membranes Made of Chitosan and Alginate. Biomacromolecules 2013, 14 (5), 1653-1660.

(25) Silva, J. M.; Caridade, S. G.; Oliveira, N. M.; Reis, R. L.; Mano, J. F. Chitosan-alginate multilayered films with gradients of physicochemical cues. J. Mater. Chem. B 2015, 3 (22), 4555-4568.

(26) Silva, J. M.; Duarte, A. R. C.; Custódio, C. A.; Sher, P.; Neto, A. I.; Pinho, A. C. M.; Fonseca, J.; Reis, R. L.; Mano, J. F. Nanostructured Hollow Tubes Based on Chitosan and Alginate Multilayers. Adv. Healthcare Mater. 2014, 3 (3), 433-440.

(27) Silva, J. M.; Georgi, N.; Costa, R.; Sher, P.; Reis, R. L.; Van Blitterswijk, C. A.; Karperien, M.; Mano, J. F. Nanostructured 3D Constructs Based on Chitosan and Chondroitin Sulphate Multilayers for Cartilage Tissue Engineering. PLoS One 2013, 8 (2), e55451.

(28) Sher, P.; Custódio, C. A.; Mano, J. F. Layer-By-Layer Technique for Producing Porous Nanostructured 3D Constructs Using Moldable Freeform Assembly of Spherical Templates. Small 2010, 6 (23), 2644-2648.
(29) Liu, Y.; Wang, A.; Claus, R. O. Layer-by-layer electrostatic selfassembly of nanoscale $\mathrm{Fe}[$ sub 3] O[sub 4] particles and. Appl. Phys. Lett. 1997, 71 (16), 2265.

(30) Silva, J. M.; Duarte, A. R. C.; Caridade, S. G.; Picart, C.; Reis, R. L.; Mano, J. F. Tailored Freestanding Multilayered Membranes Based on Chitosan and Alginate. Biomacromolecules 2014, 15 (10), 38173826.

(31) Caridade, S. G.; Monge, C.; Almodóvar, J.; Guillot, R.; Lavaud, J.; Josserand, V.; Coll, J.-L.; Mano, J. F.; Picart, C. Myoconductive and osteoinductive free-standing polysaccharide membranes. Acta Biomater. 2015, 15 (0), 139-145.

(32) Ciuti, G.; Donlin, R.; Valdastri, P.; Arezzo, A.; Menciassi, A.; Morino, M.; Dario, P. Robotic versus manual control in magnetic steering of an endoscopic capsule. Endoscopy 2010, 42 (2), 148-152.

(33) Taccola, S.; Desii, A.; Pensabene, V.; Fujie, T.; Saito, A.; Takeoka, S.; Dario, P.; Menciassi, A.; Mattoli, V. Free-Standing Poly(llactic acid) Nanofilms Loaded with Superparamagnetic Nanoparticles. Langmuir 2011, 27 (9), 5589-5595.

(34) Costa, R. R.; Castro, E.; Arias, F. J.; Rodríguez-Cabello, J. C.; Mano, J. F. Multifunctional Compartmentalized Capsules with a Hierarchical Organization from the Nano to the Macro Scales. Biomacromolecules 2013, 14 (7), 2403-2410.

(35) Mamedov, A. A.; Kotov, N. A. Free-Standing Layer-by-Layer Assembled Films of Magnetite Nanoparticles. Langmuir 2000, 16 (13), 5530-5533.

(36) Chaoyang, J.; Markutsya, S.; Pikus, Y.; Tsukruk, V. V. Freely suspended nanocomposite membranes as highly sensitive sensors. Nat. Mater. 2004, 3 (10), 721-728.

(37) Gupta, A. K.; Gupta, M. Synthesis and surface engineering of iron oxide nanoparticles for biomedical applications. Biomaterials 2005, 26 (18), 3995-4021.

(38) Sinibaldi, E.; Pensabene, V.; Taccola, S.; Palagi, S.; Menciassi, A.; Dario, P.; Mattoli, V. Magnetic Nanofilms for Biomedical Applications. J. Nanotechnol. Eng. Med. 2010, 1 (2), 021008-021008.

(39) Gil, S.; Mano, J. F. Magnetic composite biomaterials for tissue engineering. Biomater. Sci. 2014, 2 (6), 812-818.

(40) Chaubaroux, C.; Vrana, E.; Debry, C.; Schaaf, P.; Senger, B.; Voegel, J.-C.; Haikel, Y.; Ringwald, C.; Hemmerlé, J.; Lavalle, P.; Boulmedais, F. Collagen-Based Fibrillar Multilayer Films Cross-Linked by a Natural Agent. Biomacromolecules 2012, 13 (7), 2128-2135.

(41) Gaudière, F.; Morin-Grognet, S.; Bidault, L.; Lembré, P.; Pauthe, E.; Vannier, J.-P.; Atmani, H.; Ladam, G.; Labat, B. GenipinCross-Linked Layer-by-Layer Assemblies: Biocompatible Microenvironments To Direct Bone Cell Fate. Biomacromolecules 2014, 15 (5), $1602-1611$.

(42) Gil, S.; Correia, C. R.; Mano, J. F. Magnetically Labeled Cells with Surface-Modified Fe3O4 Spherical and Rod-Shaped Magnetic Nanoparticles for Tissue Engineering Applications. Adv. Healthcare Mater. 2015, 4 (6), 883-891.

(43) Mi, F.-L. Synthesis and Characterization of a Novel ChitosanGelatin Bioconjugate with Fluorescence Emission. Biomacromolecules 2005, 6 (2), 975-987.

(44) Chen, H.; Ouyang, W.; Lawuyi, B.; Martoni, C.; Prakash, S. Reaction of chitosan with genipin and its fluorogenic attributes for potential microcapsule membrane characterization. J. Biomed. Mater. Res., Part A 2005, 75A (4), 917-927.

(45) An, Q.; Nie, K.; Zhang, Y.; Wang, Y.; Hu, Y.; Dutschk, V.; Luan, $\mathrm{X}$. PAH/DAS covalently cross-linked layer-by-layer multilayers: a "nano-net" superstratum immobilizes nanoparticles and remains permeable to small molecules. Soft Matter 2015, 11 (34), 6859-6865.

(46) Li, Q.; Ge, L.; Wan, W.; Jiang, J.; Zhong, W.; Xing, M.; Ouyang, J. Magnetically Guided Fabrication of Multilayered Iron Oxide/ Polycaprolactone/Gelatin Nanofibrous Structures for Tissue Engineering and Theranostic Application. Tissue Eng., Part C 2015, DOI: $10.1089 /$ ten.tec.2015.0051.

(47) Wei, Y.; Zhang, X.; Song, Y.; Han, B.; Hu, X.; Wang, X.; Lin, Y.; Deng, $X$. Magnetic biodegradable $\mathrm{Fe} 3 \mathrm{O} 4 / \mathrm{CS} / \mathrm{PVA}$ nanofibrous membranes for bone regeneration. Biomed. Mater. 2011, 6 (5), 055008 . 
(48) Kumar, C. S. S. R.; Mohammad, F. Magnetic nanomaterials for hyperthermia-based therapy and controlled drug delivery. Adv. Drug Delivery Rev. 2011, 63 (9), 789-808.

(49) Sensenig, R.; Sapir, Y.; MacDonald, C.; Cohen, S.; Polyak, B. Magnetic nanoparticle-based approaches to locally target therapy and enhance tissue regeneration in vivo. Nanomedicine 2012, 7 (9), 14251442.

(50) Marins, J. A.; Soares, B. G.; Barud, H. S.; Ribeiro, S. J. L. Flexible magnetic membranes based on bacterial cellulose and its evaluation as electromagnetic interference shielding material. Mater. Sci. Eng., C 2013, 33 (7), 3994-4001.

(51) Correia, C. O.; Mano, J. F. Chitosan scaffolds with a shape memory effect induced by hydration. J. Mater. Chem. B 2014, 2 (21), $3315-3323$.

(52) Borges, J.; Caridade, S. G.; Silva, J. M.; Mano, J. F. Unraveling the Effect of the Hydration Level on the Molecular Mobility of Nanolayered Polymeric Systems. Macromol. Rapid Commun. 2015, 36 (4), 405-412.

(53) Almodóvar, J.; Place, L. W.; Gogolski, J.; Erickson, K.; Kipper, M. J. Layer-by-Layer Assembly of Polysaccharide-Based Polyelectrolyte Multilayers: A Spectroscopic Study of Hydrophilicity, Composition, and Ion Pairing. Biomacromolecules 2011, 12 (7), 2755-2765.

(54) Crouzier, T.; Boudou, T.; Picart, C. Polysaccharide-based polyelectrolyte multilayers. Curr. Opin. Colloid Interface Sci. 2010, 15 (6), 417-426.

(55) Markutsya, S.; Jiang, C.; Pikus, Y.; Tsukruk, V. V. Freely Suspended Layer-by-Layer Nanomembranes: Testing Micromechanical Properties. Adv. Funct. Mater. 2005, 15 (5), 771-780.

(56) Ren, K.; Crouzier, T.; Roy, C.; Picart, C. Polyelectrolyte Multilayer Films of Controlled Stiffness Modulate Myoblast Cell Differentiation. Adv. Funct. Mater. 2008, 18, 1378-1389.

(57) Richert, L.; Engler, A. J.; Discher, D. E.; Picart, C. Elasticity of Native and Cross-Linked Polyelectrolyte Multilayer Films. Biomacromolecules 2004, 5 (5), 1908-1916.

(58) Schneider, A.; Vodouhê, C.; Richert, L.; Francius, G.; Le Guen, E.; Schaaf, P.; Voegel, J.-C.; Frisch, B.; Picart, C. Multifunctional Polyelectrolyte Multilayer Films: Combining Mechanical Resistance, Biodegradability, and Bioactivity. Biomacromolecules 2007, 8 (1), 139145.

(59) Semenov, O.; Malek, A.; Bittermann, A.; Vörös, J.; Zisch, A. Engineered Polyelectrolyte Multilayer Substrates for Adhesion, Proliferation, and Differentiation of Human Mesenchymal Stem Cells. Tissue Eng., Part A 2009, 15 (10), 2977-2990.

(60) Phelps, J. A.; Morisse, S.; Hindié, M.; Degat, M.-C.; Pauthe, E.; Van Tassel, P. R. Nanofilm biomaterials: localized cross-linking to optimize mechanical rigidity and bioactivity. Langmuir 2011, 27 (3), $1123-1130$ 